\title{
Monseigneur Bourget et la reconstruction de la cathédrale de Montréal
}

\section{Léon Pouliot}

Volume 17, numéro 3, décembre 1963

URI : https://id.erudit.org/iderudit/302288ar

DOI : https://doi.org/10.7202/302288ar

Aller au sommaire du numéro

Éditeur(s)

Institut d'histoire de l'Amérique française

ISSN

0035-2357 (imprimé)

1492-1383 (numérique)

Découvrir la revue

Citer cet article

Pouliot, L. (1963). Monseigneur Bourget et la reconstruction de la cathédrale de Montréal. Revue d'histoire de l'Amérique française, 17(3), 340-362.

https://doi.org/10.7202/302288ar d'utilisation que vous pouvez consulter en ligne.

https://apropos.erudit.org/fr/usagers/politique-dutilisation/ 


\title{
MONSEIGNEUR BOURGET ET LA RECONSTRUCTION DE LA CATHÉDRALE DE MONTRÉAL
}

\author{
DESTRUCTION DE LA CATHÉDRALE \\ ET PROJET DE RECONSTRUCTION
}

\author{
$-\mathrm{I}-$ \\ L'INCENDIE DU 8 JUILLET 1852
}

\begin{abstract}
Dans ses Mémoires sur Mgr Bourget, l'abbé Pierre Poulin raconte ainsi l'incendie du 8 juillet 1852 :
\end{abstract}

Dieu, dont les desseins sont impénétrables, avait permis que ce jour-là vit réunies toutes les causes qui peuvent produire le terrible ravage de l'incendie: chaleur tropicale, absence des autorités civiles et religieuses, manque d'eau dans l'aqueduc. Le maire et plusieurs échevins n'étaient pas alors en ville. L'évêque de Montréal était en tournée pastorale et le Supérieur du Séminaire se trouvait au lac des Deux-Montagnes. L'on s'était aperçu qu'il fallait nettoyer l'aqueduc, et il n'y avait pas à le remettre. Donc, ce jour-là, le réservoir était à sec. Je puis parler de divers incidents ayant rapport à cet incendie parce que je me trouvais alors à Montréal. Etant curé à Saint-Hermas, comté des Deux-Montagnes, des affaires m'avaient appelé en ville, et j'y arrivais juste au moment où l'incendie prenait naissance à 10 heures du matin. Le feu commença rue SaintLaurent, entre les rues Sainte-Catherine et Dorchester, côté nord. La cheminée d'une forge s'enflamma et mit le feu à un petit grenier à foin situé dans le voisinage. Le quartier, qui était alors en grande partie construit en bois, prit facilement feu. Comme il arrive ordinairement dans les incendies, le vent se mit de la partie. Bientôt un vaste brasier, 
s'étendant jusqu'au pied du Coteau Baron ${ }^{1}$, et à quelque distance de l'hôpital anglais ${ }^{2}$ en descendant, forma la ceinture du feu qui devait envelopper dans sa marche la moitié du quartier Saint-Laurent et la plus grande partie du quartier Saint-Jacques. Vers trois heures de l'après-midi, cette vaste partie de la ville était réduite en cendres. Le petit hospice Saint-Joseph, résidence des vieux prêtres ${ }^{3}$, l'asile de la Providence ${ }^{4}$ et la Maternité 5 restèrent seuls debout. Le soir lorsqu'on commençait à se remettre des fatigues du jour, vers 9 heures, le feu reprit dans une écurie en arrière du théâtre de Hayes. Bientôt un nouvel incendie, aussi considérable que le premier, couvrit de ruines le faubourg Québec. A partir du carré Dalhousie jusqu'au delà des distilleries de Molson, tout fût balayé, depuis le fleuve jusqu'à la rue Craig et un peu au-delà de celle-ci, en arrière du marché Papineau. L'établissement naissant des PP. Oblats ${ }^{6}$ put heureusement échapper à la conflagration ?

Le matin du 9 juillet 1,100 maisons étaient détruites et 9,000 personnes étaient sans abri. Le feu n'avait épargné ni la cathédrale, ni le nouvel évêché inauguré six mois plus tôt, en décembre 1851. Cette conflagration suivait d'un mois celle du 6 juin, que La Minerve du 8 décrivait longuement sous ce titre "Grande conflagration dans le centre du commerce à Montréal. Perte totale estimée à $\$ 200,000$. Mort déplorable de deux enfants."

Le 8 juillet, Mgr Bourget était en visite pastorale à Vaudreuil. M. Poulin fut chargé d'aller communiquer la nouvelle à l'évêque et de solliciter son retour immédiat en ville. Mgr Bourget pleura, mais sa résignation était totale: "Dieu nous Sherbrooke.

2 Le General Hospital, rue Dorchester, là où se trouve aujourd'hui l'Hôpital Saint-Charles-Borromée.

3 Angle des rues Saint-Hubert et Demontigny.

4 Angle des rues Sainte-Catherine et Saint-Hubert.

5 Angle des rues Campeau et Lagauchetière.

- L'église Saint-Pierre, rue de la Visitation.

7 Arthur SAVAÈte: Voix Canadiennes. Vers l'abime, IX: 180 et suiv. (Paris, s.d.) - Autre récit de l'incendie dans Institut de la Providence, II: 401 et ss. 
l'avait donné, Dieu nous l'ôte; que son saint Nom soit béni." Et encore: "Merci, mon Dieu, d'avoir épargné vos pauvres."

Il décide de suspendre la visite pastorale et le 9 juillet il rentrait à Montréal. Une lettre l'y attendait; sensibles à son malheur, les religieuses de l'Hôtel-Dieu, situé alors rue SaintPaul lui offraient l'hospitalité. Des liens très intimes le rattachaient à cette communauté, c'est là qu'il avait vécu avec $\mathrm{Mgr}$ Lartigue de 1821 à 1825; c'est dans la chapelle de l'hôpital qu'il avait reçu l'onction sacerdotale, le 30 novembre 1822. Mais plus fort que les sentiments d'amitié et de reconnaissance envers une famille religieuse, est le sens du devoir chez Mgr Bourget. La conception qu'il a de son devoir lui demande de s'établir dans le quartier éprouvé, de souffrir avec ceux qui souffrent. Il répond: "Je ne puis me rendre à une si généreuse invitation; d'ailleurs, il m'est impossible de me séparer des prêtres de l'évêché qui se sont attachés à mon sort, heureux ou malheureux 8." Jusqu'en 1855, l'Hospice Saint-Joseph lui tiendra lieu d'évêché et la chapelle de la Providence sera sa cathédrale.

La présence de Mgr Bourget en ville ne fut pas inutile. "Il s'entendit aussitôt avec le commandant des forces militaires, écrit M. Poulin, afin de procurer des tentes à tant de malheureux qui étaient sans abri. Il les fit placer sur la ferme Logan, en arrière jusqu'au pied du coteau Baron. Un comité de secours fut aussi nommé afin de pourvoir au logement des sinistrés et à leur subsistance provisoire ${ }^{9}$."

Le 11 juillet, l'évêque adressait une Pastorale à la ville de Montréal. Parfaitement soumis à la volonté de Dieu, il s'efforce de communiquer ses sentiments aux fidèles:

Qu'allons-nous devenir ? Comment subvenir à tant et de si grandes misères ? C'est ce qui surpasse tout calcul humain. Tout ce que nous savons, c'est que c'est Dieu qui a soufflé, du souffle de sa colère, ce feu que la main de l'homme n'a pu maîtriser. Cela nous suffit. Il a choisi pour l'allumer, ce feu dévo10 juillet.

${ }^{8}$ Annales manuscrites de l'Hôtel-Dieu, Mgr Bourget à la Supérieure,

9 Savaète, op. cit.. 183. 
rant, le moment où nos réservoirs d'eau étaient à sec. Il a lui-même, tracé à ce feu, devenu en quelque sorte intelligent, sa route, pour qu'il épargnât ceux qu'il voulait épargner, et qu'il ruinât ceux qu'il voulait ruiner. En tout cela, il est adorable.

Puisque c'est Dieu qui l'a ainsi voulu, pourquoi ne le voudrions-nous pas? Oh! oui Seigneur, nous le voulons, et de tout notre cœur. N'êtes-vous pas le maître absolu de toutes choses ? Vous avez commandé au feu de nous consumer, et il a obéi ; maintenant, vous nous commandez la soumission à ce décret rigoureux, nous sommes à vos pieds, et nous baisons avec amour votre divine main qui a déchargé sur nous un coup si terrible. Loin de nous le plus léger murmure. Nos lèvres ne s'ouvriront que pour vous bénir. Nos cœurs sont affligés, mais ils sont calmes et résignés. $O$ Sainte résignation, vous faites aujourd'hui notre unique bien ${ }^{10}$.

Résignation qui n'est pas celle du désespéré: basée sur la foi, elle engendre l'espérance. Celui qui nous a frappés peut tout relever, et il le veut. A nous de le mériter par une vie chrétienne fervente, faite surtout de charité.

L'évêque condamne ceux qui voudraient exploiter la misère du pauvre. Il met en garde contre les crises morales qui accompagnent parfois les grands malheurs: "Nous avons tout perdu, comme vous le savez; mais tout cela ne sera rien pour nous, si notre ville épiscopale, loin de se démoraliser, n'en devient que meilleure ${ }^{11}$."

Le 23 juillet, il écrit à Mgr Guigues, évêque d'Ottawa:

Nous avons tout perdu, excepté notre confiance en Dieu qui nous a frappés si justement, sans oublier toutefois qu'il était père. Cela nous remplit de force et de courage; et nous espérons sortir du chaos, créer de nouveau de rien, comme le furent autrefois le ciel et la terre ${ }^{12}$.

Le gouvernement, le conseil de ville, la charité publique allaient venir au secours des quartiers éprouvés. Mais la recons-

10 Mand. du diocèse de Montréal, 2: 237-38.

11 Ibid.: 239.

12 AAM, Lettres de Mgr Bourget, 7: 352-353. 
truction de la cathédrale et du palais épiscopal restaient à la charge de l'évêque. L'heure eût été mal choisie pour lancer une campagne de souscription. Aussi l'évêque attendit trois mois avant d'attirer l'attention de ses fidèles sur ses propres malheurs et sur les besoins de la corporation épiscopale ${ }^{13}$.

Or, pendant ce temps, le diocèse de Saint-Hyacinthe était érigé, ce qui enlevait à Montréal un tiers de ses paroisses les plus riches. Et il paraissait normal que le diocèse naissant pensât à établir solidement son évêque avant de voler au secours de Montréal.

Avec un désintéressement admirable il y exhortait les catholiques de Saint-Hyacinthe dans la lettre même où il les remettait à la sollicitude de Mgr Jean-Charles Prince, ci-devant coadjuteur de Montréal ${ }^{14}$. Cette Pastorale allait être réimprimée plus tard pour stimuler la reconstruction de la cathédrale de Montréal. Mais, en ce moment, l'évêque oublie ses propres malheurs et ses propres besoins.

Il convient, écrit-il, que l'évêque de Saint-Hyacinthe, ait, dès la première heure, une cathédrale qui fasse honneur à la religion; et il développe en autant de paragraphes, les points suivants: une cathédrale est, dans un diocèse, une cuvre fondamentale, une œuvre catholique, une cuvre honorable, une œuvre nécessaire, enfin, une œuvre facile; il suffit, en effet, que chaque famille donne $\$ 1.00$ pendant quatre ans, et le grand objectif sera atteint.

Le 4 août, il avait écrit à Mgr Turgeon:

Nos pauvres incendiés nous paraissent sentir leur malheur plus vivement aujourd'hui que le jour de l'incendie. Toutefois leur résignation est la même. On ne leur parle guère des ruines qui nous entourent que les larmes ne se mettent à couler. Mais en même temps, le c'est Dieu qui l'a voulu est sur leurs lèvres, et l'on voit sensiblement qu'il est dans leur cœur.

13 Mand., 2: 258-270. Pastorale sur le grand incendie du 8 juillet.

14 Mand., 2: 249-258. Pastorale sur l'érection du diocèse de SaintHyacinthe et la translation de Mgr J.-C. Prince à ce nouveau siège épiscopal, 29 sept. 
Ce qui aggrave leurs maux, c'est que les protestants insultent à leur malheur "A quoi vous servent vos prières," leur disent-ils ? "Vous voyez que nous sommes plus heureux que vous." Le scandale du mystère de la Providence, qui châtie pour corriger, ne laisse pas d'embrouiller des esprits faibles et de faire mauvaise impression. V.G. voudra bien prier pour eux et pour moi, qui suis le Pasteur d'un troupeau désolé; et avec cela, tenté, au milieu d'une si grande désolation, de toutes manières ${ }^{15}$.

La souffrance de Mgr Bourget est immense; elle ne le quitte pas un instant. Il pleure avec ceux qui pleurent. Par la parole et par l'exemple, il prêche la soumission et la confiance à l'adorable volonté de Dieu. "Jusqu'à l'automne avancé, lit-on dans l'Institut de la Providence, la nouvelle demeure de l'évêque fut entourée de tentes et d'autres abris occupés par les victimes du désastre. Tous les jours, Sa Grandeur les réunissait à la chapelle de la Providence pour la prière du soir, leur adressait des paroles consolantes et leur donnait de paternels avis ${ }^{16}$.

Si préoccupé qu'il soit par les conséquences de l'incendie, Mgr Bourget ne néglige pas les autres devoirs de sa charge pastorale. En voici une liste incomplète.

Le corps de Mgr Lartigue reposait dans la cathédrale détruite le 8 juillet. Il convenait de lui donner une sépulture plus honorable: la translation des restes au caveau de l'HôtelDieu a lieu le 25 juillet. La cérémonie est présidée par Mgr Bourget et elle se déroule selon l'ordre qu'il avait lui-même rédigé jusque dans le détail ${ }^{17}$. Il écrivait à cette occasion:

Ce qu'il y a de singulier, c'est que je suis aujourd'hui ce qu'il était, il y a trente ans, c'est-à-dire sans église et dans une communauté religieuse. Ceci me donne beaucoup de courage et je me trouve heureux de pouvoir lui ressembler en quelque chose ${ }^{18}$.

${ }_{15}$ AAM. Lettres de Mgr Bourget, 7: 354-357.

16 II : 419.

17 AAM, Mgr Bourget, Lettres pers. 1850-1855; "Ordre du jour, 25 juillet 1852". Ecriture de Mgr Bourget.

18 AAM, Lettres, 7: 361-364. A Mgr Magloire Blanchet, évêque de Nesqually, 2 août 1852 . 
Le 18 août, il adresse à M. Désaulniers, du Séminaire de Saint-Hyacinthe, des "Règles pour assurer le saint voyage que vous faites en Europe et en Asie avec le jeune (Rodrigue) Masson". Il faut nous ramener le jeune seigneur encore plus saint que savant, écrit-il ${ }^{19}$. La retraite sacerdotale a lieu du 26 août au 2 septembre et elle est suivie d'une assemblée de la Société de Tempérance ${ }^{20}$. Le 22 septembre, Mgr Prince et M. Joseph Larocque revenaient de Rome, porteurs des décrets érigeant les diocèses de Saint-Hyacinthe ainsi que l'approbation du Saint-Siège au premier concile de Québec. Le soir même, il les accompagne à Québec, afin d'aviser avec l'archevêque au mode d'exécution de ces volontés de Rome.

La Lettre Pastorale sur l'érection du Diocèse de SaintHyacinthe et la translation de Mgr $J$. C. Prince à ce nouveau siège épiscopal porte la date du 29 septembre ${ }^{21}$. Le 8 octobre, il adresse au diocèse de Montréal une Pastorale touchante sur l'incendie du 8 juillet ${ }^{22}$.

Le 16 octobre, il préside à l'église de Bonsecours, le premier départ missionnaire des Sœurs de la Providence qui, répondant au désir de Mgr A.-M. Blanchet, se rendent en Orégon; messe, sermon, recommandations, lecture des obédiences, conversations intimes du Père avec ses Filles. Il revient à l'Hospice SaintJoseph à quatre heures ${ }^{23}$ et il repart presque aussitôt. Où va-t-il ? Aux Trois-Rivières où il prononcera le 18 le sermon de circonstance au sacre de Mgr Thomas Cooke.

Depuis la fin de mai, il suit de près l'organisation de l'Hôpital Saint-Patrice, destiné aux catholiques de langue anglaise. Il tient à ce que l'institution soit gouvernée selon les lois de l'Église et non selon le nationalisme outrancier des Irlandais ${ }^{24}$. Le 17 novembre, il adresse au clergé des règles qui obtiendront au jubilé le maximum de rendement ${ }^{25}$.

19 AAM, Lettres de Mgr Bourget, 7 : 388-390.

20 Mand., 2 : 242-245; 246-249.

21 Ibid.: $249-258$.

22 Ibid.: 258-270.

23 Les Scurs de la Providence au Chili, 33-36.

24 AAM, Lettres de Mgr Bourget, 7: 472-475. AM.Dowd. 8 nov. 1852.

25 Mand., 2 : 271-275. 
Le même jour, il écrit à Mgr Prince :

"J'ai commencé à rédiger une Pastorale pour publier les décrets du concile de Québec, et je passe plusieurs jours de suite sans pouvoir écrire une seule ligne ${ }^{26}$." A la fin du mois, il assiste à la consécration de la cathédrale d'Albany: "Puissé-je faire un peu de bien aux Canadiens de là-bas", écrit-il. Le 1er décembre, il officie pontificalement à la messe des funérailles du grand patriote Ludger Duvernay. Dix jours plus tard, il adresse un mot d'encouragement à une mère de famille fortement émue par l'épreuve: "Je me fais, comme de raison, un devoir de répondre aux personnes qui ont recours à moi. Mon temps est à tout le monde, ainsi que ma personne, qui se doit à tout le troupeau ${ }^{27}$."

Un aumônier est comme pris de découragement, parce que les religieuses sont gênées avec lui. Il reçoit cette réponse: "Je trouve tous les jours des religieuses qui sont très gênées avec moi ; et j'ai grand soin de les faire remonter par d'autres. Je ne finirais pas, si je vous disais là-dessus tout ce que je sais ${ }^{28}$."

Tel était Mgr Bourget et telle la conception qu'il se faisait de son devoir. Son grand amour, c'était le diocèse de Montréal. Il ne négligeait rien pour en assurer le progrès temporel et spirituel. Il ne se bornait pas au bien général et commun du diocèse. Il se penchait sur le sort malheureux des individus, parce qu'il se devait "à tout le troupeau". Il oubliait ses propres malheurs et ses propres besoins pour ne penser qu'aux autres. On était si habitué à le voir donner et se donner, à le voir se contenter de peu pour le logement, le vivre et le couvert qu'on ne se demandait pas s'il avait le nécessaire. En cette fin d'année 1852, les victimes du 8 juillet sont convenablement logées et protégées contre les froids de l'hiver; elles ont de quoi vivre. L'évêque et son personnel sont à l'étroit à l'Hospice Saint-Joseph et on ne voit pas le jour où la petite chapelle de la Providence sera remplacée par une cathédrale digne du diocèse de Montréal. Quelle est en tout cela la responsabilité de Mgr Bourget?

${ }^{26}$ A AM, Lettres de Mgr Bourget, 7: 483-485.

27 Ibid.: 519. A Mme Bondy-Tranchemontagne, à Berthier, 11 déc. déc.

28 Ibid.: 534-535. A M. Chabot, aumônier des Sœurs à Longueuil, 18 


\section{LA RECONSTRUCTION DE LA CATHÉDRALE : OŨ ET COMMENT ? UN DÉMARRAGE DIFFICILE}

La Minerve du 20 juillet publiait la note suivante:

On vient de nous communiquer un projet qui nous sourit beaucoup, et qui sourira de même, nous n'en doutons pas à tous ceux qui s'intéressent à l'évêché de Montréal. Il est question de rebâtir la cathédrale au haut de la rue St-Denis, à main droite, sur le côteau St-Louis ${ }^{29}$, un des plus beaux sites qu'on puisse trouver en cette cité pour un pareil objet. On nous informe que l'hon. D.B. Viger et M. Chs. Lacroix offrent de donner une partie du terrain et que plusieurs citoyens sont prêts à souscrire la somme nécessaire pour acheter d'un citoyen d'une autre croyance un lot de terre qui se trouve précisément sur le site choisi pour la cathédrale. Nous sommes certain que la générosité de tous ces messieurs sera récompensée par le succès de ce projet. La générosité est une manière bien élégante d'exprimer la volonté et le désir des citoyens et nous croyons que Mgr l'Evêque n'y sera pas insensible. Nous reviendrons sur ce sujet.

En effet, quatre jours plus tard, La Minerve plaide la même cause.

Elle, (la cathédrale) serait admirablement située dans cet endroit, d'abord pour le coup d'œil qui embrasse toute la cité et notre verdoyante montagne, d'un côté, et les deux rives du fleuve sur une longue distance, de l'autre; et ensuite pour être protégée contre le feu, les rues ayant là quatre-vingt pieds de large. Tout le monde comprend ici que cet endroit sera dans peu d'années un centre de population considérable.

Et l'idée semble faire son chemin, car on lit dans le même journal, à la date du 5 août:

Nous apprenons que si Mgr consent au transport de la cathédrale et de tout son établissement sur le coteau Saint-Louis, à quelques arpents de sa posi- 
tion actuelle, non seulement tout le terrain nécessaire sera mis gratis à sa disposition, mais encore les fonds requis pour le transport des matériaux restants des anciens édifices. Le coteau SaintLouis ${ }^{29}$ se trouvant à proximité des carrières et du sable et le sol étant extrêmement propice, les travaux de construction sont là des plus faciles.

Le rêve de La Minerve était beau, trop beau pour devenir une réalité. De l'angle des rues Sainte-Catherine et Saint-Denis au site qu'occupe actuellement l'institution des Sourdes-Muettes, la distance n'était pas grande, il est vrai. Mais le coteau Baron n'était pas le quartier Saint-Jacques; et les habitants du quartier Saint-Jacques croyaient avoir un droit inaliénable sur la cathédrale et l'évêché de Montréal. Ils ne tardent pas à le faire savoir dans les colonnes de La Minerve. Afin de sortir d'une inquiétude qui les irrite et les humilie, ils présentent une requête à $\mathrm{Mgr}$ Bourget, le 9 août. La réponse de l'évêque n'était pas de nature à les rassurer:

Messieurs, j'accepte avec plaisir la requête que vous venez de me présenter; je ne suis pas préparé à vous répondre d'une manière définitive, mais soyez certains que je prendrai votre demande en ma plus sérieuse considération; il y a là certainement de pressants motifs qui pourront aider ma détermination. Soyez persuadés que si je n'écoutais que la voix de mon cœur, je ne m'éloignerais point de vous, je préférerais ne point quitter mon ancienne demeure. Mais je suis homme public, et comme tel, je dois chercher l'avantage du plus grand nombre. Toutefois, croyez que les intérêts pécuniaires ne seront pour rien dans ma décision, mais que je n'envisagerai que votre avantage et le bien qui pourrait en résulter pour la ville ${ }^{30}$.

Réponse prudente et qui indique déjà où va la pensée de Mgr Bourget. Sans trop savoir lui-même quel sera le site de la cathédrale, il élimine le quartier Saint-Jacques. Pourquoi ? et comment en est-il venu là ?

29 On ne comprend pas ce que vient faire ici le coteau Saint-Louis. C'est du coteau Baron qu'il s'agit.

${ }^{30} \mathrm{La}$ Minerve, 30 août 1852. 
En se plaçant au point de vue du bien général du diocèse, Mgr Bourget avait, et dès la première heure, considéré la destruction de la cathédrale comme une occasion providentielle de doter la ville du temple majestueux que réclamaient les temps nouveaux. Autant il était indifférent à tout ce qui regardait sa personne, autant il était jaloux de l'honneur et de la gloire de l'Église. Dans sa pensée, la cathédrale doit-être l'église la plus belle et la plus imposante du diocèse; toutes proportions gardées, elle doit être pour celui-ci ce qu'est Saint-Pierre de Rome pour l’Église universelle.

Or, le terrain sur lequel s'élevait la cathédrale est trop étroit pour y ériger un temple aux vastes dimensions et vraiment digne du diocèse de Montréal. Dans la ville, la cathédrale était déjà éclipsée par la belle et grande église Notre-Dame; plusieurs paroisses de la campagne possèdent également des églises qui surpassent en beauté et en majesté l'antique cathédrale. Cependant Mgr Bourget n'a communiqué son dessein à personne, il y a simple coïncidence entre celui-ci et le vœu formulé par La Minerve.

La mauvaise humeur du quartier Saint-Jacques, la prétention qu'il affichait d'être le siège de la cathédrale et de l'évêché n'étaient pas sans embarrasser Mgr Bourget. L'esprit de clocher allait jusqu'à soupçonner l'évêque de sentiments dont il était littéralement incapable, comme le démontrait toute sa vie; s'il délaissait le quartier Saint-Jacques, disait-on, c'est qu'il préférait les riches et les grands aux pauvres et aux petits ! Quelle souffrance morale pour Mgr Bourget que le spectre de la division s'insinuant de nouveau dans la catholique population de Montréal ! Et cela ouvertement, au vu et au su des protestants qui eux, ne craignent pas de faire beau et grand quand il s'agit d'élever un temple au Seigneur.

Quand l'évêque de Montréal était convaincu que tel était son devoir, il ne se laissait pas ébranler par les mouvements d'opposition. Il attendait dans la prière l'heure de Dieu. En ce moment Montréal a besoin de toutes ses énergies, de toute sa bonne volonté pour se relever de ses ruines. Il serait malsain, 
dommageable au bien commun d'agiter le problème de la cathédrale; il restera en veilleuse. De plus, il était conforme au tempérament de Mgr Bourget de penser au bonheur des autres avant de penser au sien, de vouloir que les familles éprouvées par l'incendie puissent affronter l'hiver dans des habitations convenables et non sous les tentes, que l'on avait hâtivement dressées au lendemain du 9 juillet.

Et c'est ainsi qu'à la fin de l'année, rien n'a été fait, rien n'a été décidé relativement à la cathédrale. Le 31 décembre, La Minerve publie un communiqué de l'évêché. Mgr Bourget n'est pas en mesure de recevoir les visites qui sont d'usage au premier de l'an. Et le rédacteur commente: les citoyens "regretteront d'être privés de cet avantage, nous en sommes convaincus, mais ils devront surtout regretter la cause qui les en prive, et songer qu'il est urgent d'exécuter au plus tôt les projets que l'on a formés pour l'existence d'une nouvelle résidence convenable pour l'évêque de cette grande cité. Le fait que Sa Grandeur n'est pas en mesure de les recevoir cette année, à cause de l'exiguïté de sa résidence actuelle, rendra cette nécessité encore plus pressante."

$$
\text { - III - }
$$

\section{VERS UNE SOLUTION}

Pendant que les autres oublient ou paraissent oublier, Mgr Bourget, lui, n'oublie pas. Il se regarde toujours comme tenu en conscience de reconstruire la cathédrale et l'évêché; mais sur un autre site et avec des dimensions plus vastes, majestueuses. Mais la chose est-elle financièrement possible ? Il en suppute les chances. S'il s'agissait tout simplement de reconstruire l'église Saint-Jacques, les moyens ne manqueraient pas. Mais ce serait sacrifier le bien commun du diocèse et l'honneur de l'Église au bien particulier et à l'attachement sentimental d'un quartier urbain. Cela, il ne le peut pas. Il ne peut pas laisser à ses successeurs une situation humiliante et indigne du diocèse de Montréal. La décision qu'il prend aujourd'hui doit pouvoir répondre aux besoins de demain. Et comme c'est lui qui prend la décision, c'est lui qui doit en payer le prix. Les pensées qui 
se pressent dans l'esprit de Mgr Bourget, il nous les a livrées dans son mandement du 19 mars 1853. Les voici :

Depuis le huit juillet que nous sommes en face des tristes ruines de Notre Cathédrale et de Notre Evêché, Nous nous occupons en nous-même des moyens à prendre pour les relever. Mais tous nos calculs n'ont servi jusqu'ici qu'à Nous convaincre de notre impuissance à réparer les désastres du terrible incendie qui, en quelques heures a réduit en cendres des propriétés valant quarante mille louis courant. Depuis ce déplorable évènement, Nous sommes sans Eglise pour remplir nos fonctions épiscopales, et Nous n'avons pour nous abriter qu'un Hospice de charité. Oh! elles ne sont plus joyeuses pour nous nos grandes solennités !

Notre premier devoir a été de bénir Celui qui Nous a ôté tout ce qu'Il Nous avait donné; et notre unique consolation a été de baiser avec amour la main paternelle qui Nous frappait. Il Nous reste maintenant à travailler de toutes nos forces à réparer les désastres d'un si grand incendie. Toutes ressources humaines nous manquent pour cela. Nous ne saurions nous endetter pour cela; car Nous savons que Nous ne pourrions point rencontrer nos engagements. Les propriétés que le feu a épargnées ne suffisent pas pour notre subsistance et l'honnête entretien des prêtres qui quittent tout pour partager nos travaux et nos malheurs. D'ailleurs que sontelles ces propriétés pour faire face aux énormes dépenses que vont entrainer les nouvelles bâtisses ? La ville, après tant de désastreux incendies, et les campagnes, après tant de mauvaises années qui se font sentir, ne Nous paraissent pas préparées à une aussi grande dépense. Toutefois, Nous ne saurions rester dans ce fâcheux état. Car Nous y voyons pour la Religion des maux incalculables. Nous comprenons, et vous comprenez comme Nous, qu'un Evêque sans cathédrale et sans maison est, pour le diocèse ce que serait pour une paroisse un curé sans église et sans presbytère. Toutefois notre confiance n'en est point abattue. Nous avons vu s'élever, comme par enchantement, la première église de St-Jacques, ce temple si justement cher à notre cœur; Nous 
avons pu suivre tous les événements que ménageait la divine Providence, pour assurer le succès d'un établissement auquel le calcul humain apposait alors le sceau de l'Impossibilité ${ }^{31}$. Nous étions à même de recevoir d'utiles leçons de dévouement et d'abandon à la Divine Providence, à l'école d'un grand Maître. Aujourd'hui que nous sommes précisément ce qu'était en mil-huit-cent-vingt-un, le Fondateur de l'épiscopat à Montréal, les souvenirs de ce temps se regravent en Nous plus avant que jamais et retrempent notre courage; ce qu'il a fait et souffert pour ériger cette modeste, mais si dévote église qu'il nous a laissée en héritage, se rajeunit dans nos pensées. Il nous ouvre aujourd'hui son bon cœur, comme si souvent il le faisait (au temps) de sa vie mortelle; et il Nous semble que sa grande âme nous apparaît pour Nous redire ce que si souvent il nous répétait, et surtout ce que si constamment nous lui vîmes faire pour l'amour de la Sainte Eglise. Il est donc là, ce Père chéri, pour nous inviter à avoir foi à un meilleur avenir; en marchant sur ses traces, Nous avons l'espoir qu'un jour nos yeux, aujourd'hui si abattus à la vue de tant de ruines, seront réjouis en les voyant remplacées par des édifices qui répondent davantage aux besoins de notre époque ${ }^{32}$.

Quel était donc ce moyen auquel rêvait l'évêque de Montréal et qu'il croyait seul capable d'apporter remède à ses malheurs ? Il l'avait communiqué à son coadjuteur, Mgr Joseph Larocque; et devant la juste stupéfaction de celui-ci, il lui écrivait, de l'Hôtel-Dieu, le 7 janvier 1853 :

Je vous développerai plus au long mon projet de courir le monde pour rebâtir St-Jacques et St-Jean (le palais épiscopal). Pour ma part je consentirais de grand coeur à passer le reste de mes jours dans les rues. Mais je voudrais bien, cher Seigneur, que vous et ceux qui vous entourent n'y fussiez pas avec moi. Aussi, suis-je bien décidé, moyennant la grâce de Dieu, de ne reculer devant aucun sacrifice, pour que mon successeur soit exempt de la grande misère de réparer mes ruines matérielles ${ }^{33}$.

31 Sur ce sujet, notre ouvrage Mgr Bourget et son Temps, I: 53-76. 32 Mand., 2: 345-347.

33 AAM, Mgr Bourget, Lettres pers. 1850-1855. 
Courir le monde en mendiant, le projet ne laisse pas de nous paraître étrange. Pourquoi Mgr Bourget s'y est-il arrêté ? Pourquoi n'a-t-il pas fait appel d'abord à la charité du diocèse avant de solliciter le secours des étrangers ? La Pastorale du 8 octobre contenait une allusion discrète aux besoins de l'évêque: "Enfin, après trois mois d'une agitation indicible, Nous commençons à respirer; mais hélas ! ce n'est que pour sentir plus vivement notre cruelle position. Toutefois oubliant encore nos propres malheurs, Nous allons vous dire ceux de nos chers enfants ${ }^{34}$."

L'allusion aux malheurs et aux besoins de l'évêque n'a pas été comprise; aucune voix autorisée ne s'est fait entendre, aucun organisme n'a été formé pour relever la cathédrale de ses ruines. Une pareille indifférence au bien commun n'est pas sans nous étonner. Elle s'explique, croyons-nous, par l'impopularité du changement de site si fortement désiré par Mgr Bourget. Sans doute, les fidèles ne manquent pas qui reconnaissent à l'évêque le droit de construire sa cathédrale, où il le veut. Mais ils ne sont ni les plus nombreux, ni les plus influents. La majorité des fidèles est sentimentalement attachée au site de l'ancienne cathédrale. Et précisément parce que son opposition, disons même son hostilité à toute innovation est d'ordre sentimental, la discussion publique serait plus nuisible qu'utile. Les bons amis de Mgr Bourget se taisent, ils ne bougent pas, afin de ne pas jeter de l'huile sur le feu. Quant à lui, pendant 15 ans (1821-1836), il a trop souffert de la division des esprits et des cœurs dans la ville épiscopale, il en a trop mesuré les conséquences douloureuses pour en provoquer la répétition ${ }^{35}$. Puisque les laïques n'osent pas se compromettre, il n'ose pas, lui, intervenir personnellement. Par ailleurs, la reconstruction de la cathédrale et de l'évêché reste pour lui un problème de conscience, et elle ne doit pas souffrir de délai.

Quant à l'idée d'aller quêter en Europe, elle a aussi son explication. Dans ses voyages précédents, on lui avait spontané-

34 Mand, 2: 259.

35 Mgr Bourget et son temps, I: 77-107. 
ment offert des aumônes; il les avait refusées, parce que, pensaitil, le diocèse de Montréal est assez riche pour subvenir à ses besoins. Mais aujourd'hui, la situation n'est plus la même.

Cependant, au début de 1853 le projet de Mgr Bourget n'est plus sans appel. Il écrit à Mgr Larocque, le 8 janvier: "Je suis si peu attaché à mon opinion que j'en passerai aveuglément par celle des personnes qu'il vous plaira de consulter, par celle surtout du chapitre qui doit avoir grâce d'état pour cela ${ }^{36}$." Le 24 janvier, il écrit à $M$. Choiselat, trésorier de la Propagation de la Foi à Paris :

Jusqu'ici je n'ai rien demandé pour moi à mes diocésains. La nécessité et le bien de la religion me font néanmoins songer sérieusement à relever les tristes ruines de ma modeste cathédrale et les autres édifices épiscopaux. Mais voyez combien j'ai besoin de me remettre entre les mains de la Providence. Les édifices que l'incendie a dévorés étaient encore grevés d'une dette de t3000. Mon clergé est épuisé par ses souscriptions antérieures. $\mathrm{Ma}$ ville est appauvrie par l'incendie. Je compte sur le dévouement du clergé et sur la bonne volonté des fidèles. Mais je ne crois pas que le diocèse suffise à la dépense que nécessite la reconstruction de ces édifices. Dieu y pourvoira et sa Providence me viendra en aide ${ }^{37}$.

Ce texte n'est pas d'un homme qui serait comme pris de panique. L'évêque compte surtout sur la Providence; mais il compte aussi sur le dévoument du clergé et des fidèles.

Mais déjà les chanoines ont pris l'affaire en main. Ils cherchent et ils trouvent un moyen capable, pensent-ils, d'obtenir le but que poursuit Mgr Bourget sans que celui-ci ait à parcourir le monde en mendiant. La lettre que M. Truteau adresse aux archiprêtres, le 17 janvier 1853 nous livre le résultat de leurs délibérations.

En vertu d'un indult apporté de Rome par Mgr Prince, il était permis aux évêques du Canada de percevoir une dîme sur les

${ }^{36}$ AAM, Mgr Bourget, Lettres pers. 1850-1855.

37 AAM, Lettres, $7: 567-570$. 
revenus des curés. Les évêques avaient renoncé pour le moment à l'exercice de ce privilège. Mais les chanoines crurent que la condition de Mgr Bourget était particulière et qu'elle devait recevoir un traitement particulier. En conséquence, dans une supplique adressée à leur évêque, les curés l'inviteraient à percevoir la dîme des revenus curiaux comme le lui permettait le Saint-Siège. En prenant ainsi les devants, le clergé pourrait plus facilement susciter la générosité des fidèles; et comme l'évêque se proposait de visiter toutes les paroisses afin de recueillir les aumônes en faveur de la cathédrale, on croyait que le problème serait ainsi heureusement résolu ${ }^{38}$.

De prime abord, on serait porté à croire que le projet du chapitre était bien conçu. En fait, il était mal accordé à la réalité ! La majorité des prêtres et les fidèles dans leur ensemble ne voyaient pas plus loin que leur clocher. Ils ignoraient les besoins de l'évêché, parce qu'ils ne leur avaient jamais été exposés. Pour subvenir à ses dépenses, la corporation épiscopale ne disposait que des revenus de l'église Saint-Jacques, des droits de chancellerie et de quelques dons bénévoles. Cela suffisait dans le cours ordinaire des choses, d'autant plus que Mgr Bourget se faisait gloire de sa pauvreté et qu'il l'imposait à son personnel.

Quand, en 1848-1849, il s'était agi d'agrandir le palais épiscopal, on avait fait appel à la charité du clergé et des paroisses, et le résultat n'avait pas répondu à l'attente. Cela, Mgr Bourget le savait, qui avait lu les réponses des curés: ceux-ci s'étaient montrés plus ou moins généreux; mais, à peu d'exceptions près, ils se déclaraient incapables de secouer l'inertie des fabriciens. L'évêque vit donc, du premier coup, que la démarche du chapitre était vouée à l'échec, qu'elle susciterait dans le clergé malaise et mécontentement. Il n'hésita pas à la désavouer. La lettre qu'il adresse à $M$. Truteau, le 21 février, nous révèle sa pensée; il reconnaît la bonne volonté des chanoines, mais il déplore les effets de leur démarche:

On murmure, on se plaint, on parle de recourir au prochain concile pour révoquer l'Indult pontifical,

${ }^{38}$ A AM, Cathédrale-Reconstruction, Dossier 1. Lettre de M. Truteau aux archiprêtres, 17 janvier 1853. 
que l'on sait bien avoir été donné sans avoir été demandé; enfin l'on verrait l'évêque décimer le clergé d'un aussi mauvais œil que l'on voit le clergé décimer le peuple. Voilà où en sont les choses autant que je puis en juger par les renseignements qui m'ont été fournis.

Il tenait au bon renom des chanoines comme à la prunelle de ses yeux. Or, leur démarche est de nature à les rendre odieux.

Vous m'avez toujours vu jusqu'ici me charger de toutes les mesures de rigueur, comme c'était mon devoir, pour donner à ceux que le ciel m'envoyait pour m'aider à porter mon fardeau tout le beau et le bon d'un ministère pacifique. Je ne puis me faire à l'idée d'agir autrement.

De plus, il ne peut accepter une solution qui le rendrait trop dépendant du clergé; pour accomplir sa mission, il a besoin de toute sa liberté. Mais alors que faire?

Tout ce que je puis dire pour le moment, c'est que Dieu a, dans ses trésors, des ressources inconnues à l'homme qui met en Lui toute sa confiance. Sans ce fonds de confiance en la divine Providence, le désastre du huit juillet est un abîme sans fonds. J'ai vu bâtir St-Jacques sans secours humain. Je le verrai rebâtir de même, c'est ce que je tâche de dire à mon cœur, quand il m'échappe par défaut de confiance filiale ${ }^{39}$.

Division des fidèles sur le site de la cathédrale, mauvais esprit qui s'insinue dans le clergé, à l'occasion du problème financier, ce sont les grandes souffrances de Mgr Bourget. Cet évêque qu'on nous présente parfois comme un esprit agressif, n'aimait rien tant que l'union des esprits et des cœurs. Il avait trop souffert des divisions qui avaient déchiré Montréal de 1821 à 1836 pour en provoquer comme un retour en 1853 . C'est à cela qu'il pense quand, le 24 mars, il écrit à Mgr François-Norbert Blanchet, archevêque d'Oregon:

Les pages les plus tristes de l'histoire ecclésiastique sont celles qui relatent les mésintelligences des mis-

${ }^{39}$ A AM, Lettres de Mgr Bourget, 7: 599-603. A M. Truteau, SaintColomban, 21 février 1853. 
sionnaires qui, avec les meilleures intentions du monde, ne pouvaient s'entendre; et qui, faute d'entente, laissaient périr, sous leurs yeux, une multitude d'âmes qu'une intime cordialité eût pu sauver. Je vous avoue que c'est ce qui m'afflige le plus ici, quand cette zizanie se répand ${ }^{40}$.

Sa lettre du 2 avril à M. Manseau, curé de Joliette, est encore plus explicite:

Ma plus grande peine n'est pas précisément le refus du clergé de me venir en aide, mais la fermentation qu'a excitée dans le diocèse cette affaire, qui pouvait se terminer tout bonnement par un non motivé, selon les impressions de chacun et son manque de moyens pour le moment ${ }^{41}$.

Est-il étonnant, après cela, qu'il soit revenu à son projet d'aller tendre la main en Europe ? Mais il se rend parfaitement compte que ce ne sera pas un voyage de plaisir :

Dans la supposition qu'on en viendrait à ce parti, je conçois que je dois me sacrifier; et partir avec mon sac, plein de confiance en la divine Providence, et avec une certaine provision de patience pour ne pas perdre le mérite des humiliations qui se doivent rencontrer sur une pareille route ${ }^{42}$.$$
-\mathrm{IV}-
$$ \\ LE MANDEMENT DU 19 MARS}

Le 16 mars, sa décision est prise, et il en informe Mgr Turgeon.

Le mandement qui communique la nouvelle au diocèse est du 19 mars, mais il n'a été lu au prône que le 3 avril. Document capital pour une meilleure connaissance de l'âme de Mgr Bourget. Il ne profère aucune plainte, aucun regret. Il constate qu'il est dans l'impuissance d'accomplir ce qu'il regarde comme un devoir

\footnotetext{
${ }^{40}$ AAM, Lettres de Mgr Bourget, $7:$ 629-633.

41 AAM, Lettres de Mgr Bourget, 8: 9.

42 AAM, Mgr Bourget, Lettres pers., 1850-1855. A M. Truteau, SaintColomban, 28 février 1853.
} 
sacré. Et quel est ce devoir sacré ? Assurer envers et contre tout la reconstruction de la cathédrale et de l'évêché ? Il n'ose le promettre ni aux autres ni à lui-même. Le devoir sacré consiste à se soumettre à la Sainte volonté de Dieu. L'évêque a longuement réfléchi, il a consulté, il a prié. Il part, parce qu'il en est intimement convaincu, telle est la volonté de Dieu. Il ne s'arrête pas à scruter les desseins de la Providence, il ne cherche pas à mesurer les succès du voyage; il prévoit des souffrances, des humiliations, des échecs, il les accepte d'avance. Il serait indigne de sa mission apostolique, il serait un obstacle au progrès du diocèse de Montréal, au salut des âmes qui lui sont confiées, s'il n'exécutait pas ce qui lui apparaît clairement comme une volonté de Dieu. Écoutons-le:

Toutes choses mûrement considérées, Nous pensons, N.T.C.F., que c'est la volonté de Dieu que nous allions solliciter des secours, partout où il Nous semblera pouvoir les trouver. Tel est le but de ce troisième voyage que Nous allons entreprendre sous peu. Déjà deux fois Nous avons traversé la mer pour aller chercher un secours d'hommes dont le besoin se faisait vivement sentir. Il n'est pas nécessaire de vous dire les bénédictions qu'il a plu à Dieu de répandre sur ces deux premiers voyages. Car vous jouissez avec délices des précieux avantages qui en sont revenus au diocèse, pour la sanctification des âmes et le progrès des sciences. Si, comme nous l'espérons de ce Dieu tout bon et tout miséricordieux, ces œuvres continuent à se développer avec le même succès, vous aurez à bénir à Jamais la divine Providence d'être l'objet si spécial de ses soins maternels ${ }^{43}$.

Maintenant, quel sera le succès de ce nouveau voyage; c'est ce que nous ne pouvons pas prévoir, et ce que Nous ne cherchons pas non plus à scruter. Accoutumé que nous sommes à Nous abandonner aveuglément au bon plaisir d'un Dieu qui, malgré notre souveraine indignité, s'est toujours plu à nous combler de ses insignes faveurs. Caché dans ce petit

${ }^{43}$ Sur le premier voyage de Mgr Bourget en Europe, voir Mgr Bourget et son temps, II : 49-140. - Nous avons brièvement rappelé les résultats de son second voyage dans Rapp. Soc. Can. d'hist. de l'Eglise cath. 1956-1957, 13-18. 
coin du diocèse (Saint-Colomban), nous éprouvons d'ineffables délices à les repasser dans l'intérieur de notre âme, et Nous nous préparons de notre mieux à y répondre fidèlement.

Nous ne nous dissimulons pas toutefois les difficultés particulières que Nous présente ce nouveau voyage, à raison du personnage que Nous allons y faire. Car, il est question de solliciter, à l'étranger, des secours pécuniaires; et vous savez ce qui attend à chaque porte ceux qui tendent la main pour recevoir. Mais Nous nous considérons comme étant dans un cas si exceptionnel, que Nous n'hésitons pas de passer par dessus cette difficulté. A notre dernier voyage en Europe, l'on nous offrit quelques secours temporels : mais Nous les refusâmes, parce qu'il nous semblait alors que l'évêque d'un diocèse comme celui de Montréal, devait trouver chez lui toutes les ressources qui lui étaient nécessaires pour lui et ses œuvres. Mais aujourd'hui que Montréal est à la queue et que ses malheurs excitent une compassion générale, Nous ne croyons pas Nous déplacer en Nous mettant au nombre des victimes du terrible incendie qui Nous a mis dans la rue, et en demandant un secours dont plus que pas un, Nous avons un si pressant besoin. Ainsi, N.T.C.F., quoiqu'il en coûte beaucoup à notre pauvre nature, Nous partons avec l'intime confiance que le Seigneur Nous donnera son ange, pour Nous garder dans toutes nos voies, et Nous tracer l'itinéraire de notre voyage. Nous irons partout où il Nous inspirera d'aller et Nous frapperons à toutes les portes qu'il nous indiquera. Avant tout, Nous allons dans la Ville Sainte faire part de notre malheur au Père commun, lui rendre compte de vos âmes et recevoir sa bénédiction. Mais nous ne partirons pas sans vous dire que Nous avons droit de compter sur votre sympathie et sans faire appel à vos bons cœurs. Vous comprenez que c'est pour vous aider à vous acquitter du devoir si juste qui vous est imposé de contribuer, selon vos moyens, aux édifices religieux qui sont aux charges du diocèse, pour la raison toute simple qu'ils doivent être à l'avantage de tous les fidèles. Ce qui vous sera demandé sera peu de choses pour chacun; mais si tout le monde y met la main, Nous serons puissamment secondés 
pour faire un établissement qui réponde à la grandeur du catholicisme, dont il ne faut pas oublier l'honneur, dans une ville comme la nôtre, où nos frères séparés font, pour leurs temples tant de sacrifices. Les lugubres décombres que Nous laissons sous vos yeux vous diront plus haut et plus éloquemment que Nous ne pourrions le faire, comment vous devez vous acquitter d'un devoir si légitime. Vous entendrez leur touchant langage, et vous répondrez à leur appel. C'est le doux espoir que Nous emportons dans notre cœur et ce qui nous donnera des forces pour solliciter la charité de l'Ancien Monde en notre faveur. Et s'il vous fallait des exemples pour vous encourager à être généreux pour la reconstruction de votre cathédrale, Nous vous citerons celui des catholiques d'Albany, qui, pour la leur, viennent de fournir cinquante mille piastres. Aussi, ont-ils aujourd'hui la consolation de remplir leurs devoirs religieux dans une église qui est une merveille de notre Amérique ${ }^{44}$.

Mgr Bourget avait raison de voir grand et loin, de vouloir doter Montréal d'édifices religieux dignes d'une métropole. Il avait raison également de ne pas ajourner indéfiniment ses projets de reconstruction. Mais quand on considère la suite des événements, on n'échappe pas à l'impression qu'il y a eu erreur quelque part, que le problème a été mal engagé. Et il n'est pas facile de faire le partage des responsabilités. Pourquoi le comité de secours n'a-t-il pas affecté aux besoins de l'évêque une part des aumônes qu'il recevait ? Et que dirons-nous des fidèles $d u$ quartier Saint-Jacques ? Ils s'attribuaient sur la cathédrale des "droits" qu'ils n'avaient pas et qui étaient incompatibles avec le progrès bien entendu de l'Église de Montréal. Un mois après le désastre, ils tentent de faire reconnaître leurs "droits" par l'évêque ${ }^{45}$. La réticence de l'évêque les rend agressifs, hostiles à tout projet de reconstruction ailleurs que chez eux; ils clament à tout venant qu'une souscription qui ignorerait leur volonté est vouée à l'échec, et ils n'en sont pas autrement émus. Mgr Bourget

44 Mand., 2: 344-357.

45 A AM, Cathédrale-Reconstruction, Dossier 4. Pétition de 494 citoyens exprimant à Mgr Bourget leur désir de voir la cathédrale s'élever sur l'ancien site. Présentée le 18 août 1852. 
a-t-il exagéré le danger que présentait leur attitude ? Était-il impossible de surmonter ou de contourner la difficulté ? Il lui aurait fallu pour cela des collaborateurs actifs et habiles, mais il ne s'en est pas trouvé. Si Mgr Larocque et le chapitre ont compris, du premier coup, que le projet d'aller mendier en Europe manquait de réalisme et heurterait le sentiment populaire, ils ne présentent aucune solution de rechange qui soit valable. Bien plus, leur proposition aboutit à semer la division dans le clergé. Mais si graves qu'elles soient, ces circonstances laissent intact le devoir de l'évêque. C'est à lui qu'il incombe avant tout de reconstruire la cathédrale. Sans un acte de foi en la divine Providence, avait-il écrit, le désastre du 8 juillet est "un abime sans fond". Eh bien ! cet acte de foi sauveur, il le fera seul. Solution extrême, solution regrettable apportée à un problème qui, nous l'avons dit, avait été mal engagé.

Quelle qu'ait été ici l'erreur de jugement de Mgr Bourget, on ne peut qu'admirer son attachement à son devoir, tel que le lui présentent sa science et sa conscience, la droiture de ses intentions, la générosité avec laquelle il se sacrifie pour le bien commun. Un évêque qui s'identifie à ce point avec son Église peut se tromper parfois, il n'en reste pas moins un grand évêque.

Mais rassurons-nous; il ne partira pas avec son sac de mendiant sur le dos. Comment cela se passera-t-il ?

(à suivre)

LÉON POULIOT, S.J. 